\title{
Plumbagin induces apoptosis via the p53 pathway and generation of reactive oxygen species in human osteosarcoma cells
}

\author{
LINQIANG TIAN, DELONG YIN, YE REN, CHEN GONG, ANMIN CHEN and FENG-JIN GUO \\ Department of Orthopedics, Tongji Hospital, Tongji Medical College, \\ Huazhong University of Science and Technology, Wuhan 430030, P.R. China
}

Received July 15, 2011; Accepted October 5, 2011

DOI: $10.3892 / \mathrm{mmr} .2011 .624$

\begin{abstract}
Osteosarcoma, which is the most common primary bone tumor, occurs most frequently in adolescents. A number of studies have indicated that plumbagin (PL) (5-hydroxy-2methyl-1, 4-naphthoquinone), a compound found in the plants of the Plumbaginaceae and Droseraceae families, possesses anticancer activity. However, its anticancer effects and mechanisms against osteosarcoma have not been explored. To determine the anticancer effect of PL on osteosarcoma cell lines MG-63 and U2OS, cell viability, apoptosis, cell cycle distribution, caspase-3 and caspase-9 activity and intracellular reactive oxygen species (ROS) generation were measured, and Western blot analyses were performed. PL significantly inhibited the growth of osteosarcoma cells, particularly U2OS cells. PL up-regulated the expression of p53 in U2OS cells and p21 in the two osteosarcoma cell lines causing cell cycle arrest by decreasing the expression of murine double minute 2 (MDM2)/ cyclin B1 and cyclin D1. Furthermore, PL altered the ratio of $\mathrm{Bax} / \mathrm{Bcl}-2$, and may have triggered the mitochondrial apoptotic pathway, resulting in caspase- 3 and caspase-9 activation. We also found that PL induced the generation of ROS in osteosarcoma cell lines. To conclude, PL exerted anticancer activity on osteosarcoma cells by inducing pro-apoptotic signaling and modulating the intracellular ROS that causes induction of apoptosis. These effects may relate to the p53 status.
\end{abstract}

\section{Introduction}

Osteosarcoma is the most commonly diagnosed primary malignant bone tumor (1). Among individuals younger than 20 years, the osteosarcoma incidence rate is 8.7 per million, and the risk is higher in males (2). The most common locations of malignant tumors are regions with rapid bone growth.

Correspondence to: Dr Feng-Jin Guo, Department of Orthopedics, Tongji Hospital, Tongji Medical College, Huazhong University of Science and Technology, Wuhan 430030, P.R. China

E-mail: chenguo8889@yeah.net

Key words: plumbagin, human osteosarcoma cell line, cell cycle arrest, P53, murine double minute 2, apoptosis
The current treatment for osteosarcoma is neoadjuvant chemotherapy prior to surgery, surgical resection and chemotherapy again following surgery. With these management strategies, the 10-year disease-free survival is approximately $60 \%$ among patients with non-metastatic disease and there is a worse outcome for patients with metastatic disease at diagnosis (3). Thus, the development of new therapies is critical for improving prognosis of patients with osteosarcoma.

Plumbagin (PL; 5-hydroxy-2-methyl-1, 4-naphthoquinone), a compound found in the plants of the Plumbaginaceae and Droseraceae families, has been traditionally used for treating a number of diseases, including microbial infections and allergic reactions, in India and China (4-6). Previous studies have demonstrated that PL exhibits anti-Haemonchus contortus (7), anti-bacterial (8), hypolipidaemic and anti-atherosclerotic (9), anti-inflammation (10) and anticancer effects $(11,12)$. The molecular mechanisms of the anticancer effects of PL include inactivition of $\mathrm{NF}-\kappa \mathrm{B}$ and $\mathrm{Bcl}-2(13,14)$, disruption of the microtubule network (15), breakage of DNA (16), generation of reactive oxygen species $(17,18)$ and cell cycle arrest $(19,21)$. PL was also found to improve the radiation effect in the treatment of mouse tumor xenograft models $(22,23)$. Furthermore, PL was found to have no apoptosis-inducing effect on normal prostate epithelial RWPE-1 cells, thus it could be used as a chemotherapeutic agent (24). However, the apoptosis-inducing effect of PL on osteosarcoma cells and its mechanisms have rarely been reported.

The p53 pathway is a major growth-regulating pathway. p53, a tumor suppressor protein, plays a significant role in modulating the cell cycle and inducing apoptosis when DNA is damaged or as a cell response to stress in human cells (25). p53-induced cell cycle arrest and apoptosis requires transactivation of p21 and other cell cycle-related elements, such as cyclin B1 and cyclin D1. p21, as a CDK inhibitor, results in G1-, S-, G2-phase arrest when it is overexpressed (26). The p53 protein is regulated by various intracellular and extracellular stimuli. The activation of p53 leads to either cell apoptosis or cell growth arrest wholly or partly through induction of p21 (27). Under normal circumstances, inactive cyclin-dependent kinase 1 (CDK1) binding to cyclin B1, initiating its phosphorylation status, becomes an active cyclin B1-CDK1 complex. The complex translocates to the nucleus to play a role in phosphorylation. In this intricate process, accumulation of cyclin B1 to the threshold level was 
very significant as it guided the process (28). In a number of cancer cells, cyclin B1 was found to be overexpressed at the protein and mRNA levels at all phases of the cell cycle and peaked at the G2/M phase (29). Previous studies suggest that inhibition of cyclin B1 by various agents such as ionizing radiation (30), adriamycin (31) or sulforaphane (32) causes significant $\mathrm{G} 2 / \mathrm{M}$ arrest in numerous cancer cell types. Cyclin D1 is a key factor in extracellular signaling resulting in an excess of cells in the G1 phase. Its function was also found to be mediated by CDKs and histone. Genetic aberrations and overexpression of cyclin D1 have been noted in numerous types of human cancers (33).

The murine double minute 2 (MDM2) plays a key role in negatively moderating p53 activity in the nucleus and cytoplasm. In the two classic p53-dependent checkpoint pathways, which are activated by DNA damage and oncogenic mutation, p53 was activited by inhibiting the expression of MDM2 (34). MDM2 may inhibit the function of p53 by at least three different approaches: i) blocking p53 activity; ii) degradation of p53; and iii) exporting of p53 from the nucleus, which is the site of action. Inhibition of the expression or degradation of MDM2 induces apoptosis in tumors that retain wild-type p53 (35). It has been reported that inhibiting MDM2 expression may reactivate p53 in cancer cells, leading to their demise (35-37).

Reactive oxygen species (ROS) cause apoptotic cell death through a number of mechanisms, such as inhibition of the activity of topoisomerase II (38) and triggering the mitochondrial apoptosis pathway (39). PL was found to induce ROS generation in various types of cancer cells, such as human promyelocytic leukemia (40), human prostate cancer (18) and human cervical cancer cells (41).

In the present study, we investigated the cell proliferation inhibition activity of PL in human osteosarcoma cell lines (MG-63 and U2OS) in vitro. We also examined the effect of PL on cell cycle distribution and apoptosis. Additionally, to explore the anticancer mechanism of PL, we also detected the cell cycle- and apoptosis-related molecules, and analyzed the generation of ROS. In conclusion, PL induces the apoptosis of osteosarcoma cells through the p53 pathway and generation of ROS.

\section{Materials and methods}

Reagents. PL, dimethyl sulfoxide (DMSO), ribonuclease A (RNase A) and propidium iodide (PI) were purchased from Sigma Chemical (St. Louis, MO, USA). Fetal bovine serum (FBS), high glucose Dulbecco's modified Eagle's medium (DMEM), streptomycin and penicillin $\mathrm{G}$ were obtained from Gibco BRL (Gaithersburg, MD, USA). The antibodies to p53, p21, MDM2, Bax, Bcl-2, cyclin B1, cyclin D1 and GAPDH were obtained from Promab (Changsha, China).

Cell lines and culture. Human osteosarcoma cell lines MG63 and U2OS were obtained from the China Center for Type Culture Collection (CCTCC). The cells were cultured with high glucose DMEM supplemented with 10\% FBS, $100 \mathrm{U} /$ $\mathrm{ml}$ of penicillin $\mathrm{G}$ and $100 \mathrm{U} / \mathrm{ml}$ of streptomycin. They were cultured at $37^{\circ} \mathrm{C}$ in a humidified incubator with $5 \% \mathrm{CO}_{2}$. All cells used in the studies were in the logarithmic phase.
Cell viability assay by $C C K-8$. Inhibition of osteosarcoma MG-63 and U2OS cell proliferation by PL was analyzed by the Cell Count Kit-8 (CCK-8 Kit, Beyotime, China) assay. The CCK-8 assay was performed according to the manufacturer's instructions. Briefly, cells were seeded in fresh 96-well culture plates $\left(1 \times 10^{3}\right.$ cells per well). Following incubation overnight, the cells were treated with $0.1 \%$ DMSO (vehicle) and various concentrations of PL $(2.5,5.0,7.5$ and $10.0 \mu \mathrm{M})$ for $24 \mathrm{~h}$. The cells were rinsed twice with fresh high-glucose DMEM. Then $110 \mu \mathrm{l} \mathrm{FBS}$-free culture medium containing $10 \mu \mathrm{l}$ CCK-8 was added to each well. Following $3 \mathrm{~h}$ of incubation, the absorbance was tested on a microtiter plate reader (ELX $800 \mathrm{UV}$, Bio-Tek Instruments, Inc.) at a measured wavelength of $450 \mathrm{~nm}$. Four parallel replicates of each sample were prepared for statistical accountability. Cell viability was expressed as a percentage over the $0.1 \%$ DMSO group.

Cell cycle analysis. The cell cycle was determined by flow cytometry. MG63 and U2OS cells were seeded at a density of $3 \times 10^{5}$ cells per well and cultured overnight. We replaced the medium with fresh medium containing various concentrations of PL (2.5, 5.0, 7.5 and $10.0 \mu \mathrm{M})$ and $0.1 \%$ DMSO alone. Following $24 \mathrm{~h}$ of treatment at $37^{\circ} \mathrm{C}$, all cells were collected, washed with phosphate-buffered saline (PBS), and fixed with $70 \%$ (v/v) ice-cold ethanol for $1 \mathrm{~h}$ at $4^{\circ} \mathrm{C}$. The fixed cells were treated with $50 \mu \mathrm{g} / \mathrm{ml}$ RNase A and $50 \mu \mathrm{g} / \mathrm{ml}$ PI for $30 \mathrm{~min}$ at room temperature. At least 10,000 cells were analyzed by flow cytometry (FACSCalibur, Becton Dickinson, USA).

Analysis of cell apoptosis by Annexin V/PI staining. To assess the development of apoptosis induced by PL, MG63 and U2OS cells were stained with Annexin V-fluorescent isothiocyanate (FITC) conjugate and PI. MG63 and U2OS cells (1x10 $)$ were cultured in 24-well plates. Following overnight incubation, these cells were treated with various concentrations of PL $(2.5,5.0,7.5$ and $10.0 \mu \mathrm{M})$ and $0.1 \%$ DMSO alone for $24 \mathrm{~h}$ and collected by trypsinization. After being twice washed with $4^{\circ} \mathrm{C}$ PBS, the cell pellets were suspended in $195 \mu \mathrm{l}$ ice-cold $1 \mathrm{X}$ binding buffer at a density of approximately $1 \times 10^{6}$ cells $/ \mathrm{ml}$, and then incubated with $5 \mu \mathrm{l}$ Annexin V-FITC (Beyotime) for $10 \mathrm{~min}$ at room temperature in the dark. After the cells were centrifugated at $1,000 \mathrm{x}$ g for $5 \mathrm{~min}, 200 \mu \mathrm{l}$ ice-cold $1 \mathrm{X}$ binding buffer containing $10 \mu \mathrm{l}$ PI was added followed by flow cytometry using the FACSCalibur flow fluorocytometer (Becton Dickinson) within $1 \mathrm{~h}$.

Measurements of caspase-3 and-9 activities. This assay is based on the ability of the active enzyme to cleave the chromophore from the enzyme substrates of caspase-9, acetyl-Leu-GluHis-Asp-p-nitroanilide (Ac-LEHD-pNA), and caspase-3, acetyl-Asp-Glu-Val-Asp-p-nitroanilide (Ac-DEVD-pNA). The hydrolysis of the peptide substrate Ac-LEHD-pNA by caspase-9 or of the peptide substrate Ac-DEVD-pNA by caspase-3 results in the release of the p-nitroaniline (pNA) moiety. pNA moiety is yellow, and the concentration may be detected by measuring the absorbance at $405 \mathrm{~nm}$ wavelength. With its capacity, we can infer the activity of caspase- 3 or caspase- 9 . Cells $\left(1 \times 10^{6}\right.$ per well) were seeded into 6-well plates, incubated overnight, and then exposed to various concentrations of PL $(2.5,5.0,7.5$ and $10.0 \mu \mathrm{M}$ ) and 0.1\% DMSO for $24 \mathrm{~h}$. Caspase-9 and caspase-3 


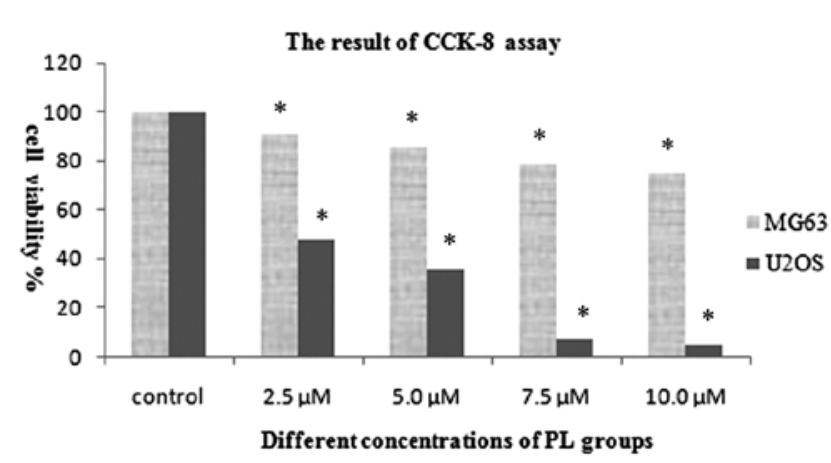

Figure 1. Effects of plumbagin (PL) on cell proliferation inhibition in osteosarcoma cells. The cell proliferative inhibition effect of PL in U2OS and MG63 cells for $24 \mathrm{~h}$. The cell growth inhibition activity of PL was assessed using the CCK- 8 Kit. Results are expressed as the percentage of cell proliferation relative to the proliferation of the control ( $0.1 \%$ DMSO). The data shown are the mean from three independent experiments. Each value is the mean of three determinations. "Significant difference between control and different concentration of plumbagin-treated cells, and U2OS and MG63 cells at the same concentration, as analyzed by one-way ANOVA and Dunnett's test $(\mathrm{P}<0.05)$.

activities were measured using the caspase-9 activity assay kit and the caspase- 3 activity assay kit (Beyotime), seperately. The release of pNA was monitored at $405 \mathrm{~nm}$ using a standard curve of defined pNA solutions. Values were expressed as nmol pNA/ $\mathrm{mg}$ of total protein.

Measurement of the intracellular ROS. Intracellular ROS generation induced by PL in MG63 and U2OS cells was measured by flow cytometry following staining with DCHF-DA according to the manufacturer's instructions (Beyotime). Briefly, cells were seeded in 6-well plates at a density of $3 \times 10^{5}$ per well, allowed to attach overnight, and were exposed to various concentrations of PL $(2.5,5.0,7.5$ and $10.0 \mu \mathrm{M}$ ) and $0.1 \%$ DMSO for $24 \mathrm{~h}$. The supernatants were replaced with $1.5 \mathrm{ml} 20 \mu \mathrm{M}$ DCHF-DA solution. Cells were washed three times with fresh FBS-free medium following incubation for $20 \mathrm{~min}$ at $37^{\circ} \mathrm{C}$. Then cells were collected and the fluorescence was measured by flow cytometry.

Western blot analysis. MG63 and U2OS cells (1x10 $10^{6}$ per dish) were seeded in $6 \mathrm{~cm}$ dishes. Following incubation overnight, the cells were treated with various concentrations of PL (2.5, 5.0, 7.5 and $10.0 \mu \mathrm{M}$ ) and 0.1\% DMSO for $24 \mathrm{~h}$. The cells were collected and lysed in RIPA buffer containing protease inhibitors (cocktail). The homogenates were centrifuged at $12,000 \mathrm{rpm}$ for $15 \mathrm{~min}$ at $4^{\circ} \mathrm{C}$ and the supernatant fraction was collected for immunoblotting. The same amounts of proteins were resolved by SDS-PAGE (6-12\%) and transferred to PVDF membranes. The membranes were then incubated with the indicated primary antibodies followed by a horseradish peroxidase (HRP) secondary antibody and developed with an enhanced chemiluminescence detection reagent (Beyotime). Grayscale analyses were measured by the Gel Doc XR imaging system (Bio-Rad, USA), and the software was Quantity One (version 4.6).

Statistical analysis. The results are expressed as the means \pm standard errors obtained from at least 3 separate experi-

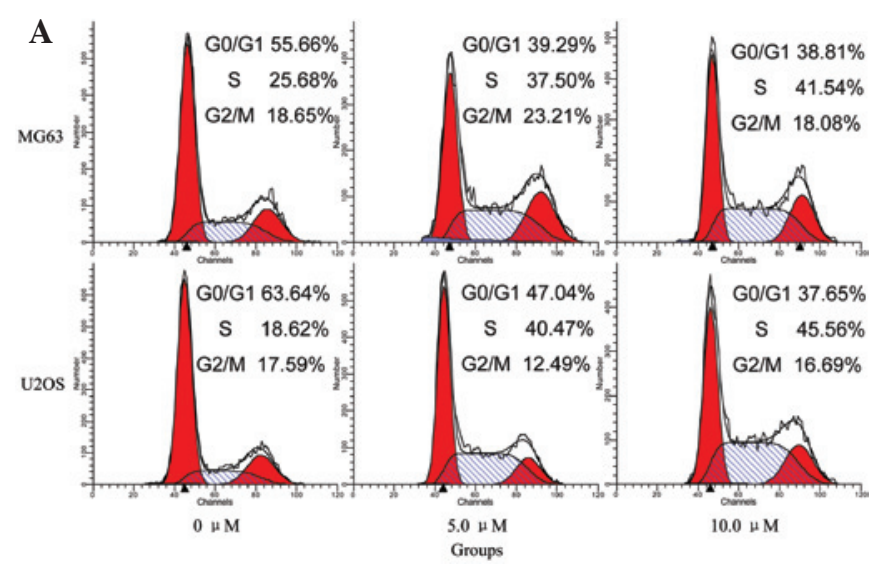

B

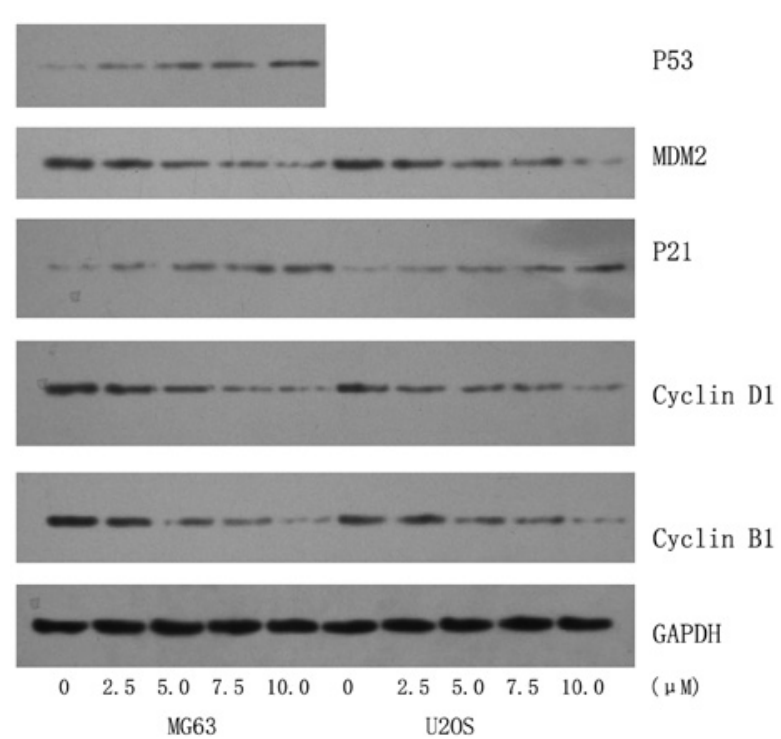

Figure 2. Effect of plumbagin (PL) on the regulation of cell cycle arrest. (A) The distribution of the cell cycle in PL-treated osteosarcoma cell lines. (B) The expression of cell cycle-related factors in PL-treated osteosarcoma cell lines.

ments. Statistical comparisons of the results between MG63 and U2OS cell lines by one-way ANOVA and between groups in MG63 or U2OS cell lines were performed by independent t-test. $\mathrm{P}<0.05$ was considered to be statistically significant. All data analysis was carried out using SPSS 15.0 software.

\section{Results}

PL inhibits cell proliferation in MG63 and U2OS cells. To study the growth inhibition ability of PL in osteosarcoma, we first detected the effects of PL on MG63 and U2OS cell lines. As shown in Fig. 1, PL inhibited the proliferation of the two cell lines in a dose-dependent manner. In U2OS cells, cell proliferation was reduced by more than $60 \%$ with $5 \mu \mathrm{M}$ and by more than $90 \%$ with $10 \mu \mathrm{M}$ treatment at $24 \mathrm{~h}$. In MG63 cells, the inhibition was approximately 15 and $25.3 \%$ at the same PL concentrations at $24 \mathrm{~h}$, seperately. The difference between the cell lines and various groups in the same cell lines was significant $(\mathrm{P}<0.05)$. The concentrations of PL that were chosen were based on previous publications involving other types of tumor cells and a rat model (13,41-44). 
A
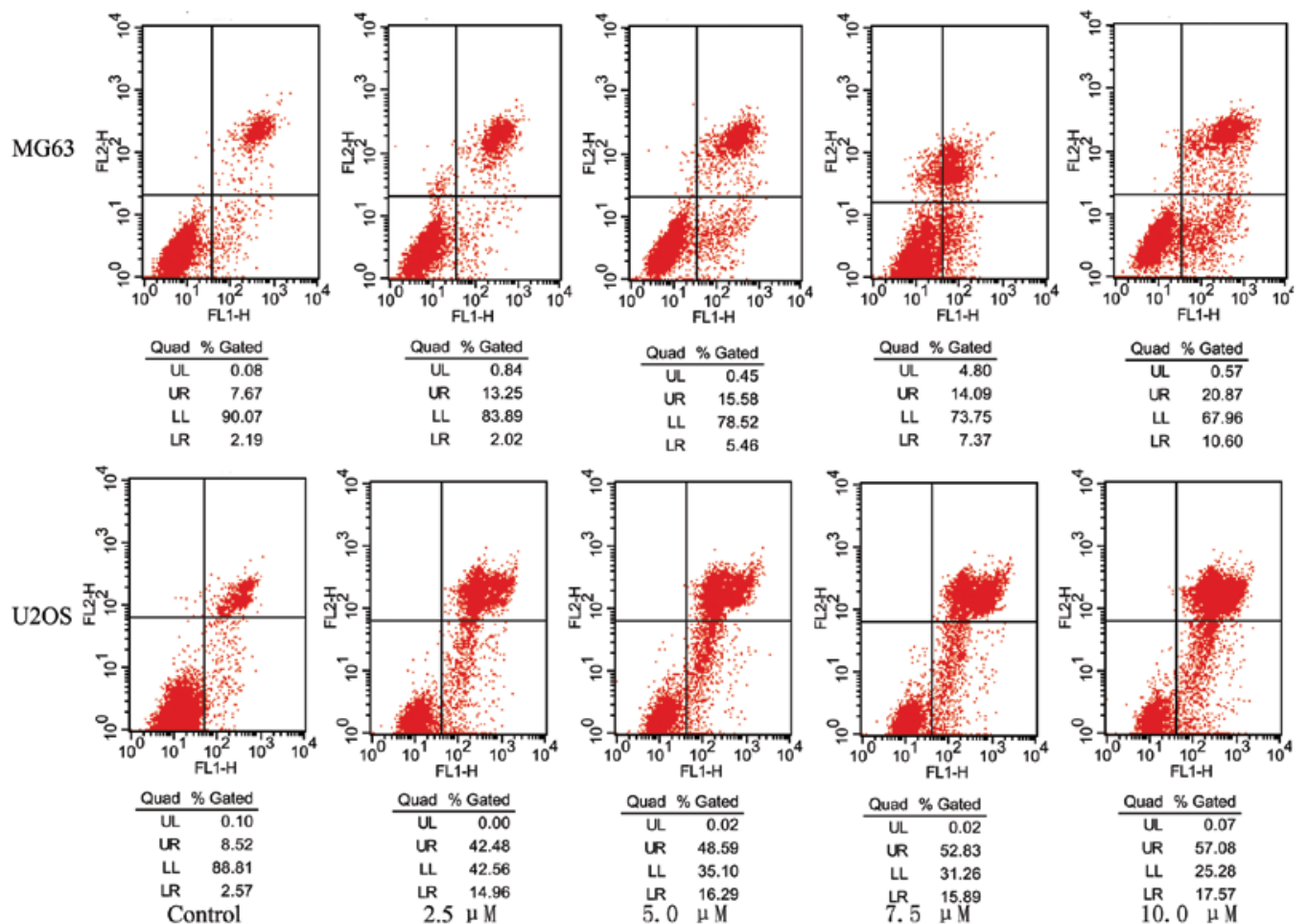

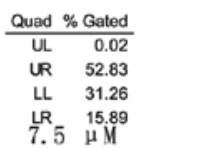

T.5 $\quad \begin{array}{ll}15.89 \\ 4 M \mathrm{M}\end{array}$

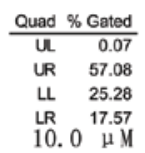

B
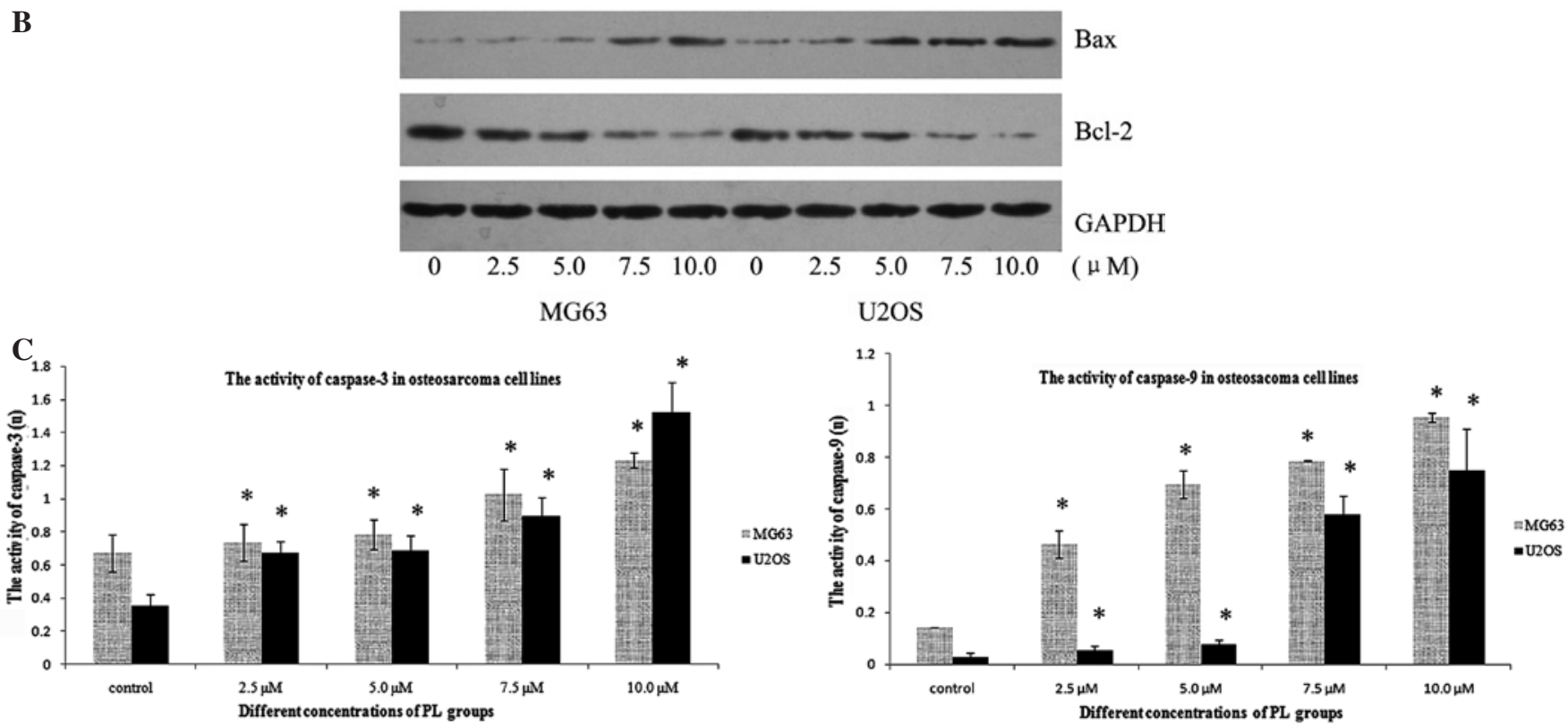

Figure 3. Plumbagin (PL) induced apoptosis through the initiation of the mitochondrial pathway. (A) Quantitative evaluations of Annexin V/PI assay by flow cytometry. Cells were treated with various concentrations of PL for $24 \mathrm{~h}$ and then apoptotic cells were examined by flow cytometry. (B) The expression level of Bcl-2/Bax proteins. The Bcl-2 and Bax expression levels of PL-treated osteosarcoma cells were determined by Western blot analysis. (C) The activation of caspase-9 and caspase-3 in MG63/U2OS cell lines. Caspase-9 and-3 were assessed using caspase-9/3 activity assay kits. Each value is the mean of three determinations. "Significant difference between control and PL-treated cells, and U2OS and MG63 cells at the same concentration as analyzed by one-way ANOVA and Dunnett's test $(\mathrm{P}<0.05)$.

PL induces cell cycle arrest and regulates cell cycle-related proteins. To investigate the mechanism by which PL induces cell proliferation inhibition, the cell cycle distribution was evaluated by flow cytometry. The results showed that cell exposure to PL caused a markedly increased percentage of cells in the $\mathrm{S}$ phase in the two cell lines when compared with the control (Fig. 2A).
Subsequently, we evaluated the effects of PL on cell cycleregulating factors at the protein level. As shown in Fig. 2B, PL increased the expression of p53 in the U2OS cells, and p21 in the two cell lines in a dose-dependent manner. We also observed that PL decreased the expression of MDM2, cyclin B1 and cyclin D1 in a dose-dependent manner in the two cell lines. As there was no functional p53 in the MG63 
The result of ROS generation

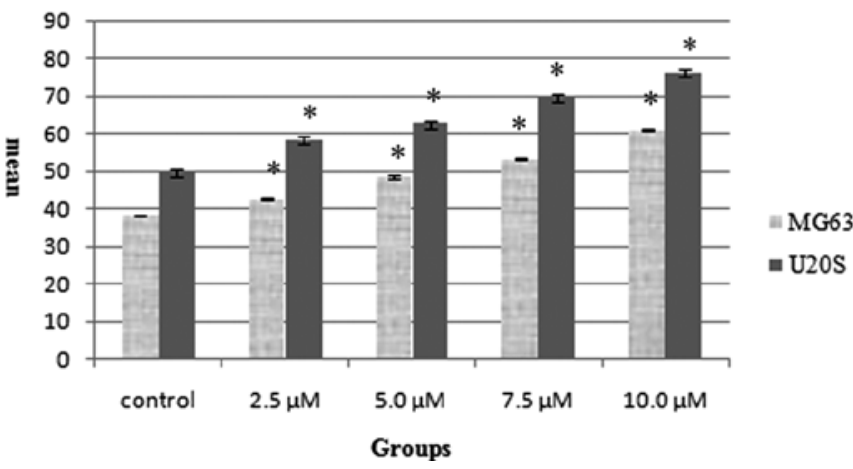

Figure 4. Plumbagin (PL) treatment causes ROS generation in U2OS and MG63 cells in a dose-dependent manner. Each value is the mean \pm SD of three determinations. "Significant difference between control and different concentration of PL-treated cells, and U2OS and MG63 cells at the same concentration, as analyzed by one-way ANOVAand Dunnett's test $(\mathrm{P}<0.05)$.

cells, the mechanisms by which PL increased p21 expression and decreased MDM2, cyclin B1 and cyclin D1 expression remain unknown. Thus, we hypothesized that other mechanisms existed, independent of p53, to regulate the cell cycle by PL.

PL induces apotosis by triggering the mitochondrial apoptosis pathway in human osteosarcoma cells. PL-induced apoptosis has been reported in a number of types of cancer cells, such as human lung cancer $(20)$, breast $(13,42)$ and prostate cancer cells $(18,24)$, but this has never been characterized in osteosarcoma cells. Therefore, we assessed the ability of PL to induce apoptosis in MG63 and U2OS cells by Annexin V/PI staining. As shown in Fig. 3A, Annexin V/ PI staining revealed that PL induced apoptotic cell death in a dose-dependent manner in the two cell lines. In U2OS cells, following incubation for $24 \mathrm{~h}$, approximately $57.4 \%$ cells underwent apoptosis when exposed to $2.5 \mu \mathrm{M}$ PL. At $10 \mu \mathrm{M}$, the apoptosis ratio reached $74.7 \%$. In MG63 cells, following the same treatment, approximately $16.2 \%$ cells underwent apoptosis when exposed to $2.5 \mu \mathrm{M}$ PL. The apoptotic ratio was approximately $32.1 \%$ at $10 \mu \mathrm{M}$. These results clearly showed that PL induces apoptosis of osteosarcoma cells, and this effect may also be related to the status of p53.

To explore whether PL induces apoptosis by triggering the mitochondrial apoptotic pathway, we measured alterations in the protein expression of the $\mathrm{Bcl}-2 / \mathrm{Bax}$ and the activities of caspase-9/3. As shown in Fig. 3B, Western blot analysis showed that treatment with PL increased the Bax protein level and decreased the Bcl-2 protein level in osteosarcoma cells. This phenomenon was gradually pronounced with the increasing concentration of PL, which led to an increase in the pro-apoptotic/anti-apoptotic ratio, resulting in cell apoptosis. As shown in Fig. 3C, the activity of caspase-3 and-9 increased significantly compared with the control group.

$P L$ increases the generation of ROS. Recent studies have revealed that ROS serve as significant signaling intermediates in apoptosis induction by various natural compounds including diallyl trisulfide, isothiocyanates and guggulsterone $(45,46)$.
The induction of apoptosis by PL in a human cervical cancer cell line was also correlated with ROS generation (41). We assumed that PL induces apoptosis in osteosarcoma cell lines by generating ROS. Therefore, we determined the ROS generation in MG63 and U2OS cells following incubation with various concentrations of PL. Intracellular ROS generation in PL-treated cells was monitored by flow cytometry after staining the cells with DCHF-DA, which is cleaved by esterases and oxidized in the presence of peroxides to fluorescent DCF. As shown in Fig. 4, PL-treated MG63 and U2OS cells showed a concentration-dependent increase in DCF fluorescence compared with DMSO-treated cells in the two cell lines at $24 \mathrm{~h}$. The ROS generation in U2OS cells was slightly higher than that in MG63 cells at the same concentration. The mechanism needs further exploration.

\section{Discussion}

This study novelly explored the effect of PL on human osteosarcoma cell lines U2OS and MG63 in vitro. PL inhibited cell proliferation and induced apoptosis of U2OS and MG63 cells, particularly U2OS cells. The difference between these cell lines is that U2OS has functional p53 while MG63 does not. According to this, we hypothesized that the effect of PL is related to the p53 pathway, which is consistent with previous studies suggesting that PL-induced cell cycle arrest is related to the p53 status $(13,44)$. However, this result is in contrast to a study involving human prostate cancer cells (18).

To confirm that the p53 pathway was involved, we detected the expression of p53 and its downstream factors at the protein level. We found that the expression of these factors was altered following treatment with PL (Fig. 2B). These changes were marked at $24 \mathrm{~h}$, particularly at high concentrations. These results were similar to previous studies $(13,47)$. We also found osteosarcoma cell stagnation in the $\mathrm{S}$ phase rather than the G2/M phase. This was not consistent with previous research (47). We also hypothesized that activated cyclin D1 plays a significant role in this process. We also confirm that MDM2 expression was suppressed. The result is proof that PL regulates the p53 pathway.

The mitochondrial apoptotic pathway is one of the two major apoptotic pathways, which is triggered by a series of intracellular stress, and activates caspase- 9 and its downstream caspase-3, cleaving cellular proteins. The pathway was found to be primarily regulated by Bcl-2 family proteins (48). The alteration of ratios between pro-apoptotic and anti-apoptotic family proteins may contribute to apoptosis. In the present study, osteosarcoma cells treated with PL exhibited upstream Bcl-2 expression and downstream Bax expression. We also observed that the activation of caspase- 9 and caspase- 3 was increased. These results suggest that PL induces apoptosis in osteosarcoma cells through the mitochondrial pathway.

Our data showed that PL induced ROS generation in osteosarcoma cells, and this may be correlated with cell death. ROS may change the redox state of cells leading to cell apoptosis through multiple pathways $(41,49)$. ROS may also adjust Bax expression, a phenomenon that was evident in our study. We also found that PL was capable of inducing ROS generation in osteosarcoma cell lines; yet the involved mechanism requires further elucidation. 
In the present study, PL inhibited human osteosarcoma cell proliferation, caused cell cycle arrest and induced apoptosis in MG63 and U2OS cell lines, particularly in U2OS cells. PL-induced cell cycle arrest may be correlated with up-regulated p53 expression in U2OS cells and p21 expression in the two cell lines, and decreased MDM2, cyclin D1 and cyclin B1 expression. PL-induced apoptosis may be via ROS generation and the mitochondrial pathway. PL inhibition of proliferation and induction of apoptosis may be associated with the status of p53. These results suggest that PL may be an effective chemopreventive agent against human osteosarcoma.

\section{Acknowledgements}

This study was supported by a grant from the Major State Basic Research Development Program of China (973 Program) (no. 2002CB513100).

\section{References}

1. Damron TA, Ward WG and Stewart A: Osteosarcoma, chondrosarcoma, and Ewing's sarcoma: National Cancer Data Base Report. Clin Orthop Relat Res 459: 40-47, 2007.

2. Mirabello L, Troisi RJ and Savage SA: International osteosarcoma incidence patterns in children and adolescents, middle aged and elderly persons. Int J Cancer 125: 229-234, 2009.

3. Longhi A, Errani C, De Paolis M, Mercuri M and Bacci G: Primary bone osteosarcoma in the pediatric age: state of the art. Cancer Treat Rev 32: 423-436, 2006.

4. Wang YC and Huang TL: Screening of anti-Helicobacter pylori herbs deriving from Taiwanese folk medicinal plants. FEMS Immunol Med Microbiol 43: 295-300, 2005.

5. Wang YC and Huang TL: Anti-Helicobacter pylori activity of Plumbago zeylanica L. FEMS Immunol Med Microbiol 43 : 407-412, 2005

6. Dai Y, Hou LF, Chan YP, Cheng L and But PP: Inhibition of immediate allergic reactions by ethanol extract from Plumbago zeylanica stems. Biol Pharm Bull 27: 429-432, 2004.

7. Fetterer RH and Fleming MW: Effects of plumbagin on development of the parasitic nematodes Haemonchus contortus and Ascaris suum. Comp Biochem Physiol C 100: 539-542, 1991.

8. Kuete V, Tangmouo JG, Meyer JJ and Lall N: Diospyrone, crassiflorone and plumbagin: three antimycobacterial and antigonorrhoeal naphthoquinones from two Diospyros spp. Int J Antimicrob Agents 34: 322-325, 2009.

9. Sharma I, Gusain D and Dixit VP: Hypolipidaemic and antiatherosclerotic effects of plumbagin in rabbits. Indian J Physiol Pharmacol 35: 10-14, 1991.

10. Checker R, Sharma D, Sandur SK, et al: Plumbagin inhibits proliferative and inflammatory responses of $\mathrm{T}$ cells independent of ROS generation but by modulating intracellular thiols. J Cell Biochem 110: 1082-1093, 2010.

11. Klaus V, Hartmann T, Gambini J, et al: 1,4-Naphthoquinones as inducers of oxidative damage and stress signaling in $\mathrm{HaCaT}$ human keratinocytes. Arch Biochem Biophys 496: 93-100, 2010.

12. Mandala Rayabandla SK, Aithal K, Anandam A, et al: Preparation, in vitro characterization, pharmacokinetic, and pharmacodynamic evaluation of chitosan-based plumbagin microspheres in mice bearing B16F1 melanoma. Drug Deliv 17: 103-113, 2010.

13. Ahmad A, Banerjee S, Wang Z, Kong D and Sarkar FH: Plumbagin-induced apoptosis of human breast cancer cells is mediated by inactivation of NF-kappaB and Bcl-2. J Cell Biochem 105: 1461-1471, 2008.

14. Sandur SK, Ichikawa H, Sethi G, Ahn KS and Aggarwal BB: Plumbagin (5-hydroxy-2-methyl-1,4-naphthoquinone) suppresses NF-kappaB activation and NF-kappaB-regulated gene products through modulation of p65 and IkappaBalpha kinase activation leading to potentiation of apoptosis induced by cytokine and chemotherapeutic agents. J Biol Chem 281: 17023-17033, 2006.

15. Acharya BR, Bhattacharyya B and Chakrabarti G: The natural naphthoquinone plumbagin exhibits antiproliferative activity and disrupts the microtubule network through tubulin binding. Biochemistry 47: 7838-7845, 2008.
16. Fujii N, Yamashita Y, Arima Y, Nagashima $M$ and Nakano $H$ : Induction of topoisomerase II-mediated DNA cleavage by the plant naphthoquinones plumbagin and shikonin. Antimicrob Agents Chemother 36: 2589-2594, 1992.

17. Kawiak A, Piosik J, Stasilojc G, et al: Induction of apoptosis by plumbagin through reactive oxygen species-mediated inhibition of topoisomerase II. Toxicol Appl Pharmacol 223: 267-276, 2007.

18. Powolny AA and Singh SV: Plumbagin-induced apoptosis in human prostate cancer cells is associated with modulation of cellular redox status and generation of reactive oxygen species. Pharm Res 25: 2171-2180, 2008.

19. Yang SJ, Chang SC, Wen HC, Chen CY, Liao JF and Chang CH: Plumbagin activates ERK1/2 and Akt via superoxide, Src and PI3-kinase in 3T3-L1 cells. Eur J Pharmacol 638: 21-28, 2010.

20. Shieh JM, Chiang TA, Chang WT, et al: Plumbagin inhibits TPA-induced MMP-2 and u-PA expressions by reducing binding activities of NF-kappaB and AP-1 via ERK signaling pathway in A549 human lung cancer cells. Mol Cell Biochem 335: 181-193, 2010.

21. Shih YW, Lee YC, Wu PF, Lee YB and Chiang TA: Plumbagin inhibits invasion and migration of liver cancer HepG2 cells by decreasing productions of matrix metalloproteinase- 2 and urokinase-plasminogen activator. Hepatol Res 39: 998-1009, 2009.

22. Nair S, Nair RR, Srinivas P, Srinivas G and Pillai MR: Radiosensitizing effects of plumbagin in cervical cancer cells is through modulation of apoptotic pathway. Mol Carcinog 47: 22-33, 2008.

23. Devi PU, Rao BS and Solomon FE: Effect of plumbagin on the radiation-induced cytogenetic and cell cycle changes in mouse Ehrlich ascites carcinoma in vivo. Indian J Exp Biol 36: 891-895, 1998.

24. Aziz MH, Dreckschmidt NE and Verma AK: Plumbagin, a medicinal plant-derived naphthoquinone, is a novel inhibitor of the growth and invasion of hormone-refractory prostate cancer. Cancer Res 68: 9024-9032, 2008.

25. Harris SL and Levine AJ: The p53 pathway: positive and negative feedback loops. Oncogene 24: 2899-2908, 2005.

26. Gartel AL and Radhakrishnan SK: Lost in transcription: p21 repression, mechanisms, and consequences. Cancer Res 65: 3980-3985, 2005.

27. Zolota V, Sirinian C, Melachrinou M, Symeonidis A and Bonikos DS: Expression of the regulatory cell cycle proteins p21, p27, p14, p16, p53, mdm2, and cyclin E in bone marrow biopsies with acute myeloid leukemia. Correlation with patients' survival. Pathol Res Pract 203: 199-207, 2007.

28. Porter LA and Donoghue DJ: Cyclin B1 and CDK1: nuclear localization and upstream regulators. Prog Cell Cycle Res 5: 335-347, 2003.

29. Yu M, Zhan Q and Finn OJ: Immune recognition of cyclin B1 as a tumor antigen is a result of its overexpression in human tumors that is caused by non-functional p53. Mol Immunol 38: 981-987, 2002.

30. Theron $\mathrm{T}$ and Bohm L: Cyclin B1 expression in response to abrogation of the radiation-induced G2/M block in HeLa cells. Cell Prolif 31: 49-57, 1998.

31. Manni I, Mazzaro G, Gurtner A, et al: NF-Y mediates the transcriptional inhibition of the cyclin B1, cyclin B2, and cdc25C promoters upon induced G2 arrest. J Biol Chem 276: 5570-5576, 2001.

32. Singh SV, Herman-Antosiewicz A, Singh AV, et al: Sulforaphane-induced $\mathrm{G} 2 / \mathrm{M}$ phase cell cycle arrest involves checkpoint kinase 2-mediated phosphorylation of cell division cycle 25C. J Biol Chem 279: 25813-25822, 2004.

33. Fu M, Wang C, Li Z, Sakamaki T and Pestell RG: Minireview: cyclin D1: normal and abnormal functions. Endocrinology 145: 5439-5447, 2004.

34. Zhang Y, Wolf GW, Bhat K, et al: Ribosomal protein L11 negatively regulates oncoprotein MDM2 and mediates a p53-dependent ribosomal-stress checkpoint pathway. Mol Cell Biol 23: 8902-8912, 2003

35. Dickens MP, Fitzgerald R and Fischer PM: Small-molecule inhibitors of MDM2 as new anticancer therapeutics. Semin Cancer Biol 20: 10-18, 2010.

36. Saha MN, Micallef J, Qiu L and Chang H: Pharmacological activation of the p53 pathway in haematological malignancies. J Clin Pathol 63: 204-209, 2010.

37. Zhang Y, Shi Y, Li X, et al: Inhibition of the p53-MDM2 interaction by adenovirus delivery of ribosomal protein L23 stabilizes p53 and induces cell cycle arrest and apoptosis in gastric cancer. J Gene Med 12: 147-156, 2010. 
38. Kovviok A, Piosik J, Gwizdek-Wisniewska A, Stosilojc G, Bigda $J$ and Lojkowska E: Induction of apoptosis through reactive oxygen species mediated inhibition of topoisomerase II by plumbagin from Drosera. Planta Medica 73: 806-807, 2007.

39. Kuo PL, Chen CY and Hsu YL: Isoobtusilactone A induces cell cycle arrest and apoptosis through reactive oxygen species/ apoptosis signal-regulating kinase 1 signaling pathway in human breast cancer cells. Cancer Res 67: 7406-7420, 2007.

40. Xu KH and Lu DP: Plumbagin induces ROS-mediated apoptosis in human promyelocytic leukemia cells in vivo. Leuk Res 34 658-665, 2010

41. Srinivas P, Gopinath G, Banerji A, Dinakar A and Srinivas G: Plumbagin induces reactive oxygen species, which mediate apoptosis in human cervical cancer cells. Mol Carcinog 40: 201-211, 2004.

42. Kuo PL, Hsu YL and Cho CY: Plumbagin induces G2-M arrest and autophagy by inhibiting the AKT/mammalian target of rapamycin pathway in breast cancer cells. Mol Cancer Ther 5: 3209-3221, 2006.

43. Prozialeck WC: Toxicology and Applied Pharmacology. Foreword. Toxicol Appl Pharmacol 238: 191, 2009.

44. Hsu YL, Cho CY, Kuo PL, Huang YT and Lin CC: Plumbagin (5-hydroxy-2-methyl-1,4-naphthoquinone) induces apoptosis and cell cycle arrest in A549 cells through p53 accumulation via c-Jun NH2-terminal kinase-mediated phosphorylation at serine 15 in vitro and in vivo. J Pharmacol Exp Ther 318: 484-494, 2006.
45. Xiao D, Vogel V and Singh SV: Benzyl isothiocyanate-induced apoptosis in human breast cancer cells is initiated by reactive oxygen species and regulated by Bax and Bak. Mol Cancer Ther 5: 2931-2945, 2006.

46. Kim YA, Xiao D, Xiao H, et al: Mitochondria-mediated apoptosis by diallyl trisulfide in human prostate cancer cells is associated with generation of reactive oxygen species and regulated by Bax/Bak. Mol Cancer Ther 6: 1599-1609, 2007.

47. Gomathinayagam R, Sowmyalakshmi S, Mardhatillah F, Kumar R, Akbarsha MA and Damodaran C: Anticancer mechanism of plumbagin, a natural compound, on non-small cell lung cancer cells. Anticancer Res 28: 785-792, 2008.

48. Adams JM and Cory S: The Bcl-2 apoptotic switch in cancer development and therapy. Oncogene 26: 1324-1337, 2007.

49. Wang CC, Chiang YM, Sung SC, Hsu YL, Chang JK and Kuo PL: Plumbagin induces cell cycle arrest and apoptosis through reactive oxygen species/c-Jun N-terminal kinase pathways in human melanoma A375.S2 cells. Cancer Lett 259: 82-98, 2008. 